IASSNS-HEP-95/109

\title{
Geodesics around a dislocation
}

\author{
Fernando Moraes \\ School of Natural Sciences \\ Institute for Advanced Study \\ Princeton, NJ 08540 \\ U. S. A.
}

\begin{abstract}
One method of gaining some insight into the motion of particles in a medium with topological defects (e.g., electrons in a dislocated metal) is to look at the geodesics of the medium around the defect. In this work the Hamilton-Jacobi equation for the geodesics in a continuous medium containing a torsional defect, an edge dislocation, is solved by using perturbation theory to first order in the Burgers vector.
\end{abstract}

${ }^{*}$ On leave from:

Departamento de Física

Universidade Federal de Pernambuco

50670-901 Recife, PE, Brazil 
Topological defects in solids have been described by Riemann-Cartan geometry since the early 1950's [1]. More recently, Katanaev and Volovich [2] have shown the equivalence between three-dimensional gravity with torsion and the theory of defects in solids. Based in this formalism, the nonrelativistic quantum mechanics of electrons or holes around disclinations [3, 4] and some properties of the quantum electromagnetic field also around disclinations [5, 6] have been studied. Disclinations, defects associated with the breaking of rotational symmetry, are common in lower dimensional systems like liquid crystals [7] and an important ingredient in the making of geometrically frustrated amorphous solids [8]. They do not appear in bulk crystals,though, due to the very high elastic energy involved in their formation. Dislocations, associated with translational symmetry breaking, do appear in ordinary 3D crystals, but the asymmetry the! y provoke in the medium makes it difficult to study dynamical properties of objects moving in their midst. By presenting the geodesics around a dislocation, this work contributes to the visualization of the dynamics of such objects and hopefully, will provide a means of obtaining some insight into the motion of particles and the geometrical optics in dislocated media.

We are concerned here with the geometrical structure of a crystalline medium that contains a single edge dislocation. An edge dislocation may be formed [2] by placing a pair of opposing parallel disclinations next to each other as a dipole (Fig. 1). Each disclination is made by either removing or inserting a wedge of material. In the continuum limit, at large distances from the defect, the medium is described by the metric [2]

$$
d s^{2}=d z^{2}+\left(1+\frac{m}{2 \pi} \frac{2 h r \sin \theta-h^{2}}{r^{2}}\right)\left(d r^{2}+r^{2} d \theta^{2}\right),
$$

in cylindrical coordinates. The wedge angles are given by $\pm m$ and the disclinations are at a distance $h$ apart. The Burgers vector $\vec{b}=-m h \hat{\mathrm{x}}$ is therefore along the $\mathrm{x}$-axis and the defect itself is along the $\mathrm{z}$-axis. Since the defect 
preserves translational symmetry along the z-axis, from now on we will be working in a $\mathrm{z}=$ constant surface.

The geodesics may be obtained by requiring that the distance between two given points in the manifold be a minimum. That is, if $\gamma$ is a curve parameterized by $t$ joining the points at $t=t_{0}$ and at $t=t_{1}$ in the manifold,

$$
d s^{2}=g_{i j} d x^{i} d x^{j}
$$

leads to

$$
s[\gamma]=\int_{t_{0}}^{t_{1}}\left(g_{i j} \dot{x}^{i} \dot{x}^{j}\right)^{1 / 2} d t .
$$

Requiring $s[\gamma]$ to be minimum, determines the path $\gamma$. The geodesic $\gamma$ is then a solution to the equation

$$
\frac{d^{2} x^{i}}{d t^{2}}+\Gamma_{j k}^{i} \frac{d x^{j}}{d t} \frac{d x^{k}}{d t}=0
$$

where the Christoffel symbols of the second kind, $\Gamma_{j k}^{i}$, are

$$
\Gamma_{j k}^{i}=\frac{1}{2} g^{i s}\left(\frac{\partial g_{s j}}{\partial x^{k}}+\frac{\partial g_{s k}}{\partial x^{j}}-\frac{\partial g_{j k}}{\partial x^{s}}\right)
$$

and $g_{i j} g^{i j}=1$.

In the language of Lagrangian mechanics, $s[\gamma]$ is the action functional and the integrand in equation (3) is the Lagrangian. This procedure, when applied to the metric (1) leads to a cumbersome pair of coupled differential equations of second order. We follow instead the Hamilton-Jacobi method described in [9]. But first we rewrite the $z=$ const. section of metric (1) in Cartesian coordinates and, since we are interested in effects far away from the defect (i.e. $r>>h$ ), neglect the term in $h^{2} / r^{2}$ :

$$
d s^{2}=\left(1-\frac{b}{\pi} \frac{y}{x^{2}+y^{2}}\right)\left(d x^{2}+d y^{2}\right) .
$$

Justified by this same argument we will perform below perturbation theory in powers of $b$. 
It is well known 10$]$ that the Newtonian-like action

$$
W[\gamma]=\frac{1}{2} \int_{t_{0}}^{t_{1}} g_{i j} \dot{x}^{i} \dot{x}^{j} d t
$$

also leads to the equations of geodesics. Using this action we proceed to write Hamilton-Jacobi equation

$$
\frac{\partial W}{\partial t}+H\left(\frac{\partial W}{\partial x^{i}}\right)=0
$$

where $H=L=\frac{1}{2} g_{i j} \dot{x}^{i} \dot{x}^{j}$ is the Hamiltonian and $L$ is the Lagrangian. Now, writing $H$ in terms of the moments $p_{i}=\frac{\partial L}{\partial \dot{x}^{i}}=\frac{\partial W}{\partial x^{i}}$ we get

$$
2\left(1-\frac{b}{\pi} \frac{y}{x^{2}+y^{2}}\right) \frac{\partial W}{\partial t}+\left(\frac{\partial W}{\partial x}\right)^{2}+\left(\frac{\partial W}{\partial y}\right)^{2}=0
$$

It follows that

$$
W=c_{1} t+\widetilde{W}(x, y)
$$

implying

$$
\left(\frac{\partial \widetilde{W}}{\partial x}\right)^{2}+\left(\frac{\partial \widetilde{W}}{\partial y}\right)^{2}+2 c_{1}\left(1-\frac{b}{\pi} \frac{y}{x^{2}+y^{2}}\right)=0
$$

Next, we write the perturbation series for $\widetilde{W}$

$$
\widetilde{W}=\widetilde{W}_{0}+b \widetilde{W}_{1}+b^{2} \widetilde{W}_{2}+\ldots
$$

Substituting (12) into (11) and equating terms of same order in $b$ we find

$$
\left(\frac{\partial \widetilde{W}_{0}}{\partial x}\right)^{2}+\left(\frac{\partial \widetilde{W}_{0}}{\partial y}\right)^{2}+2 c_{1}=0
$$

and

$$
\frac{\partial \widetilde{W}_{0}}{\partial x} \frac{\partial \widetilde{W}_{1}}{\partial x}+\frac{\partial \widetilde{W_{0}}}{\partial y} \frac{\partial \widetilde{W}_{1}}{\partial y}=\frac{c_{1}}{\pi} \frac{y}{x^{2}+y^{2}}
$$

up to first order corrections. 
Equation (13) is easily solved by the ansatz

$$
\widetilde{W}_{0}=c_{2} x+c_{3} y
$$

where the constants $c_{1}, c_{2}$ and $c_{3}$ are such that

$$
c_{1}=-\frac{1}{2}\left(c_{2}^{2}+c_{3}^{2}\right) \text {. }
$$

Now we turn to equation (14) which becomes

$$
c_{2} \frac{\partial \widetilde{W}_{1}}{\partial x}+c_{3} \frac{\partial \widetilde{W}_{1}}{\partial y}=\frac{c_{1}}{\pi} \frac{y}{x^{2}+y^{2}} .
$$

This equation is solved by the method of characteristics [1]]: first differentiate $W_{1}$ with respect to the auxiliary variable $\mathrm{u}$,

$$
\frac{d \widetilde{W}_{1}}{d u}=\left(\frac{\partial x}{\partial u}\right)\left(\frac{\partial \widetilde{W}_{1}}{\partial x}\right)+\left(\frac{\partial y}{\partial u}\right)\left(\frac{\partial \widetilde{W}_{1}}{\partial y}\right)
$$

then by comparing (17) and (18) make the identifications

$$
\frac{d \widetilde{W}_{1}}{d u}=\frac{c_{1}}{\pi} \frac{y}{x^{2}+y^{2}}, \frac{\partial x}{\partial u}=c_{2}, \frac{\partial y}{\partial u}=c_{3}
$$

It follows that

$$
x=c_{2} u+t_{1} \text {, and } y=c_{3} u,
$$

where $t_{1}$ is another auxiliary variable. Now we substitute (20) in the first equation in (19) obtaining

$$
\frac{d \widetilde{W}_{1}}{d u}=\frac{c_{1} c_{3}}{\pi} \frac{u}{\left(c_{2} u+t_{1}\right)^{2}+\left(c_{3} u\right)^{2}} .
$$

Integration with respect to $u$ of the above equation, followed by substitution of $u=y / c_{3}$ and $t_{1}=x-\frac{c_{2}}{c_{3}} y$ (from (20)) and use of (16) yields $W \approx c_{1} t+\widetilde{W}_{0}+b \widetilde{W}_{1}=-\frac{1}{2}\left(c_{2}^{2}+c_{3}^{2}\right) t+c_{2} x+c_{3} y-\frac{c_{3} b}{4 \pi} \ln \left(x^{2}+y^{2}\right)+\frac{c_{2} b}{2 \pi} \arctan \left(\frac{c_{2} x+c_{3} y}{c_{3} x-c_{2} y}\right)$. 
The corresponding solution (up to a constant $A$ ) of the equations of motion is obtained by differentiation with respect to $c_{2}$ and $c_{3}$ :

$$
-c_{2} t+x+\frac{b}{2 \pi} \arctan \left(\frac{c_{2} x+c_{3} y}{c_{3} x-c_{2} y}\right)+\frac{b}{2 \pi}\left(\frac{c_{2} c_{3}}{c_{2}^{2}+c_{3}^{2}}\right)=0
$$

and

$$
-c_{3} t+y-\frac{b}{4 \pi} \ln \left(x^{2}+y^{2}\right)-\frac{b}{2 \pi}\left(\frac{c_{2}^{2}}{c_{2}^{2}+c_{3}^{2}}\right)=0 .
$$

Inserting the missing constant, eliminating $t$ between the above equations and making the substitution $B=c_{3} / c_{2}$, we obtain

$$
y=A+B x+\frac{b}{2 \pi}\left[\frac{1}{2} \ln \left(x^{2}+y^{2}\right)+B \arctan \left(\frac{x+B y}{B x-y}\right)+1\right],
$$

which gives the correct limit in the absence of the defect $(b=0)$. A first iteration of this equation (still keeping to first order in $b$ ) allows us to plot the geodesics, which are shown in Figs. 2-4. Each figure shows a bundle of geodesics parallel to each other at infinity, approaching and being deflected by the dislocation located at the origin of the plots.

Due to the particular choice of coordinates, plotting geodesics that asymptotically are of the kind $\mathrm{x}=$ const. (i.e. vertical), is tricky. To do so, we first divide equation (25) by $B$ and then take the limit $B \rightarrow \infty$. We are left with

$$
C=x+\frac{b}{2 \pi} \arctan \left(\frac{y}{x}\right)
$$

where $C$ is constant. As before, this gives the correct vertical geodesics in the limit $b=0$. Inversion of the above equation gives

$$
y=x \tan \left[\frac{2 \pi}{b}(C-x)\right]
$$

Because of the periodicity of $\tan \theta$ we need only to choose a convenient range for $\theta=\frac{2 \pi}{b}(C-x)$ in order to plot the geodesics. Since our approach is only valid away from the origin, the range $-\pi / 2<\theta<\pi / 2$ is excluded. We use 
then the ranges $-3 \pi / 2<\theta<-\pi / 2$ and $\pi / 2<\theta<3 \pi / 2$. The result is shown in Fig. 5.

In conclusion, the solution to the equation of geodesics in a medium containing one edge dislocation has been found by using perturbation theory to first order in the Burgers vector. A single dislocation produces a dilatation that breaks the translational symmetry of the crystalline lattice causing a deformation of the geodesics around the defect as it is clear from Figs. 25. This gives an intuitive picture to what happens to (classical) electrons moving in a dislocated metal or to the propagation of rays in a dislocated continuous medium, for example.

Acknowledgment This work was partially supported by CNPq.

\section{References}

[1] E. Kröner, Continuum theory of defects, in "Les Houches, Session XXXV, 1980 - Physics of Defects" (R. Balian et al., Ed.), pp. 282-315, North-Holland, Amsterdam, 1981.

[2] M. O. Katanaev and I. V. Volovich, Ann. Phys. (NY) 216 (1992) 1.

[3] Claudio Furtado and Fernando Moraes, Phys. Lett. A 188 (1994) 394.

[4] C. Furtado, B.G.C. da Cunha, F. Moraes, E.R. Bezerra de Mello and V.B. Bezerra, Phys. Lett. A 195 (1994) 90.

[5] Fernando Moraes, Phys. Lett. A 204 (1995) 399.

[6] Fernando Moraes, Mod. Phys. Lett. A 10 (1995) 2335.

[7] M. J. Bowick, L. Chandar, E.A. Schiff and A. M. Srivastava, Science 263 (1994) 943.

[8] M. Kleman, Adv. Phys. 38 (1989) 605. 
[9] Stanislaw L. Bażański, J. Math. Phys. 30 (1989) 1018.

[10] C.W. Misner, K.S. Thorne and J.A. Wheeler, Gravitation (Freeman, San Francisco, 1973).

[11] Daniel Zwillinger, Handbook of differential equations (Academic Press, San Diego, 1992).

\section{Figure Captions}

Figure 1: Disclination dipole making up an edge dislocation.

Figure 2: Geodesics around edge dislocation, $B=-1$.

Figure 3: Geodesics around edge dislocation, $B=0$.

Figure 4: Geodesics around edge dislocation, $B=1$.

Figure 5: Geodesics around edge dislocation, $B=\infty$. 
Fig. 1

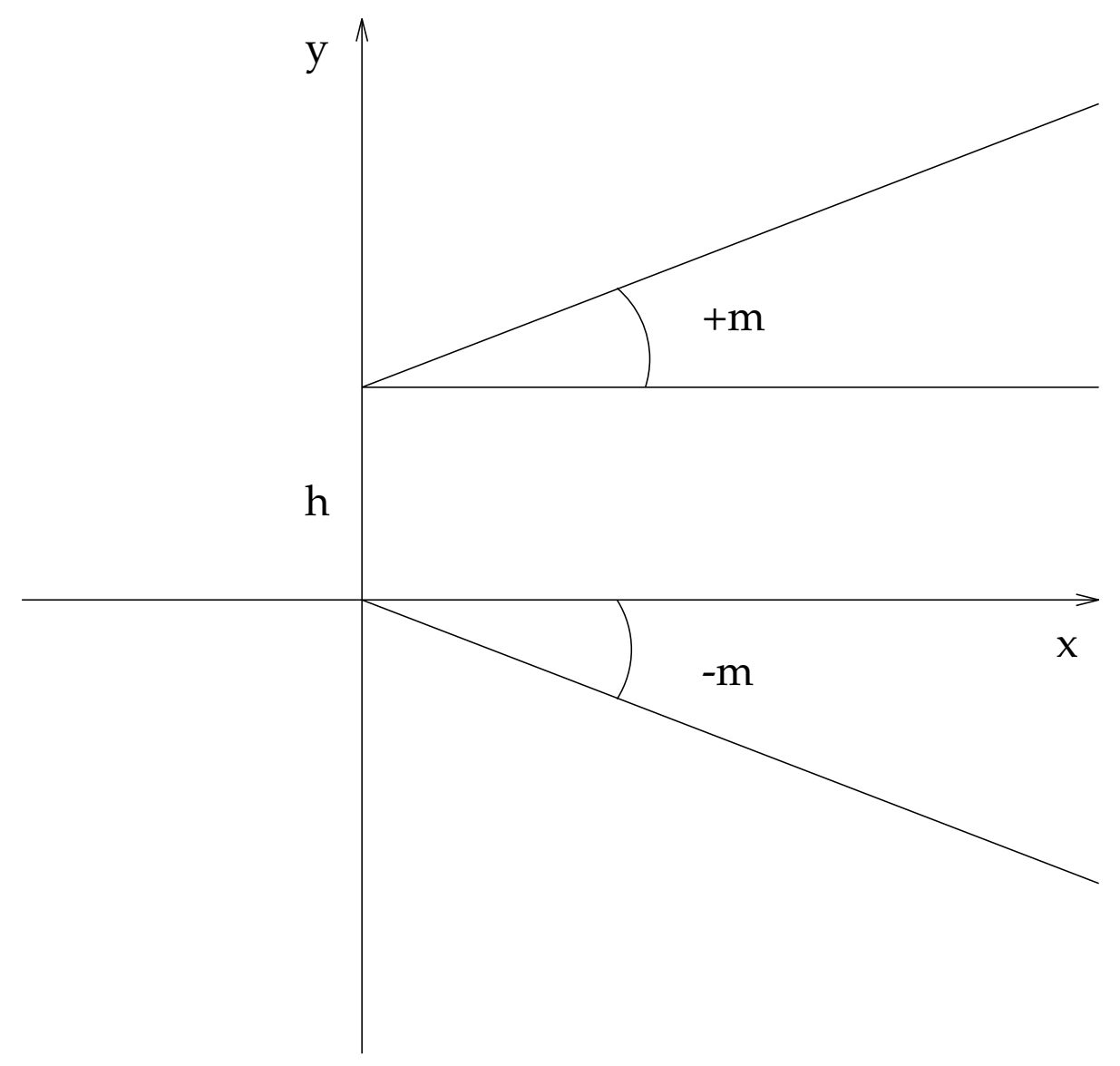




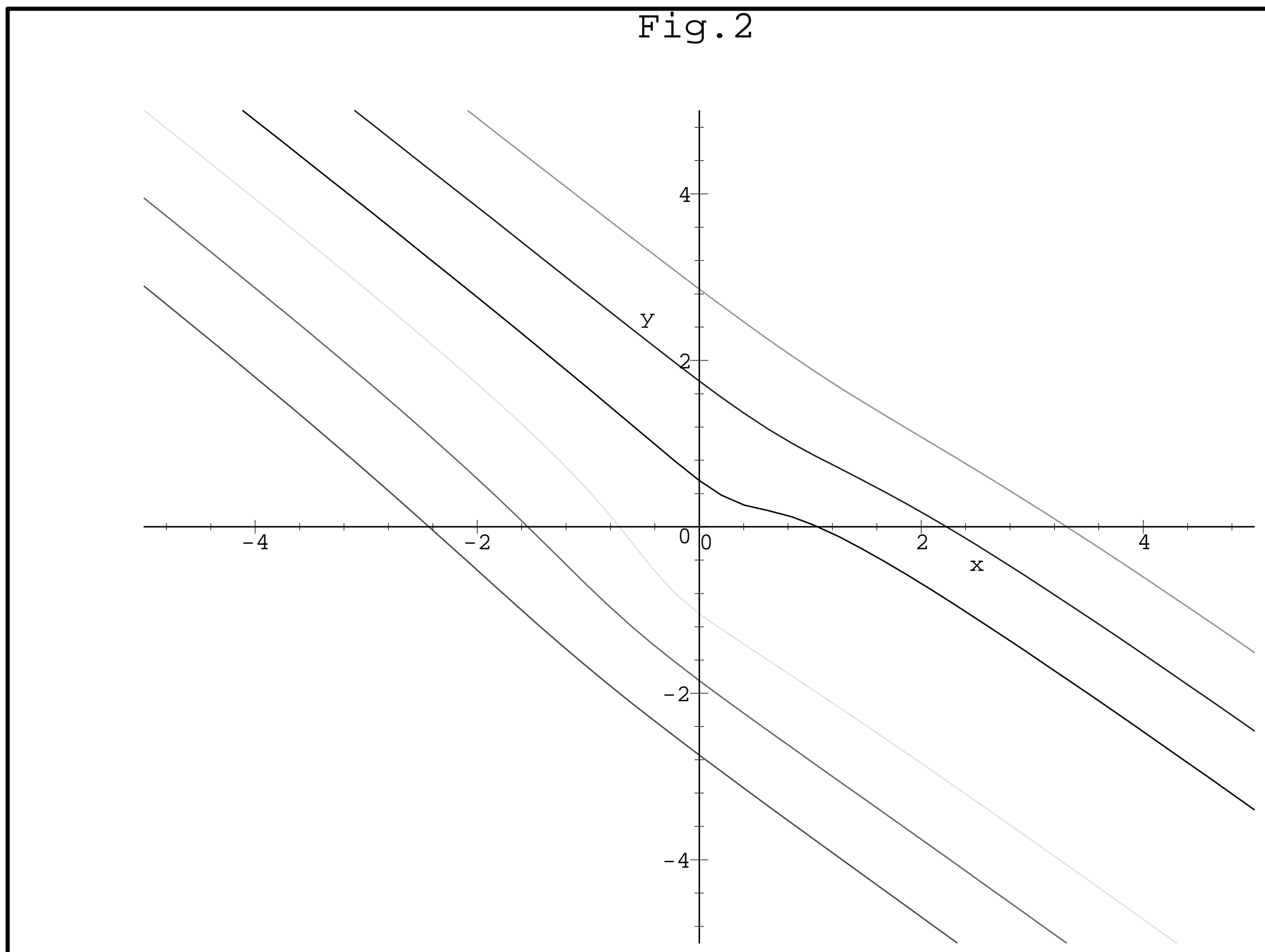




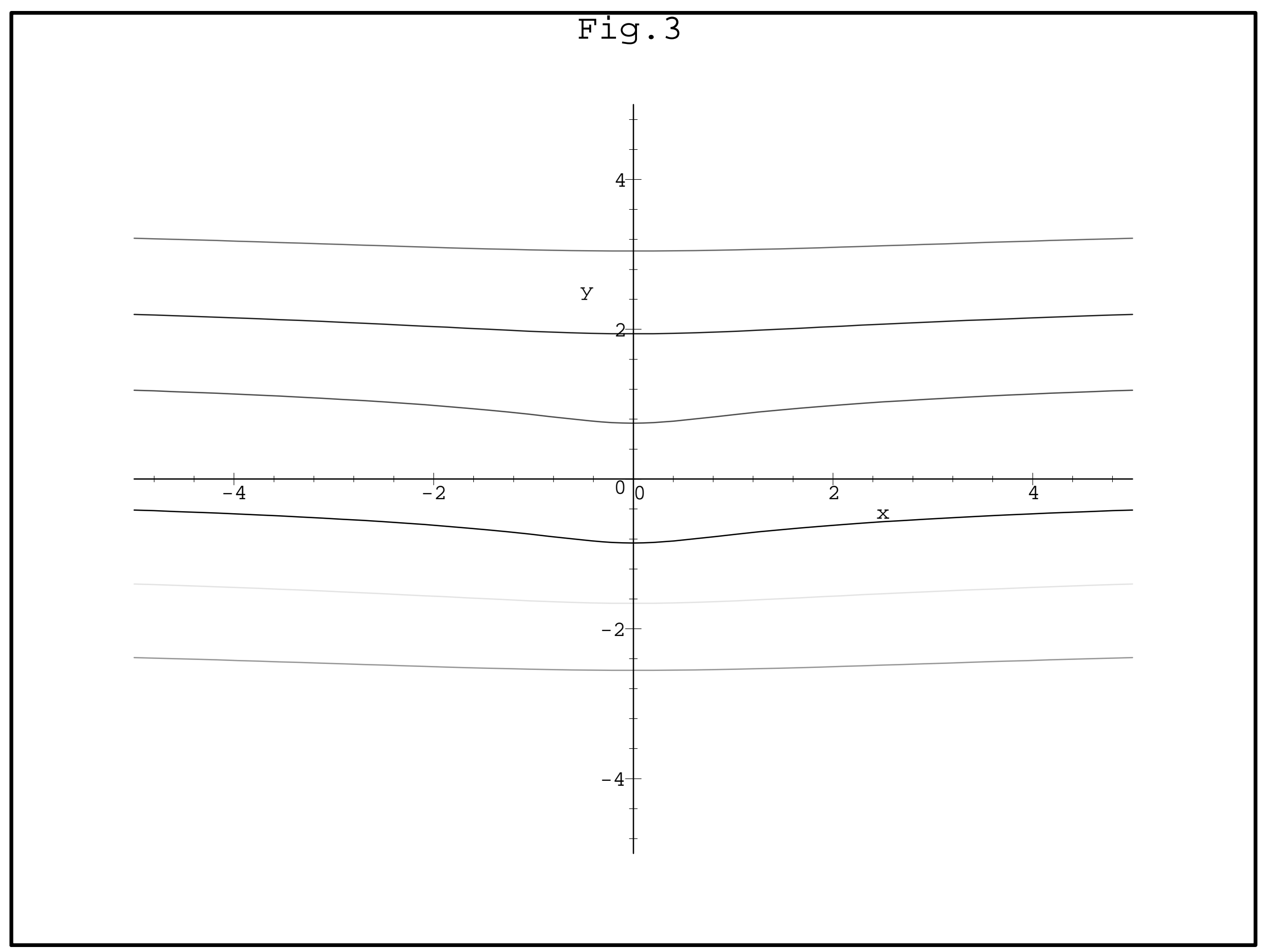




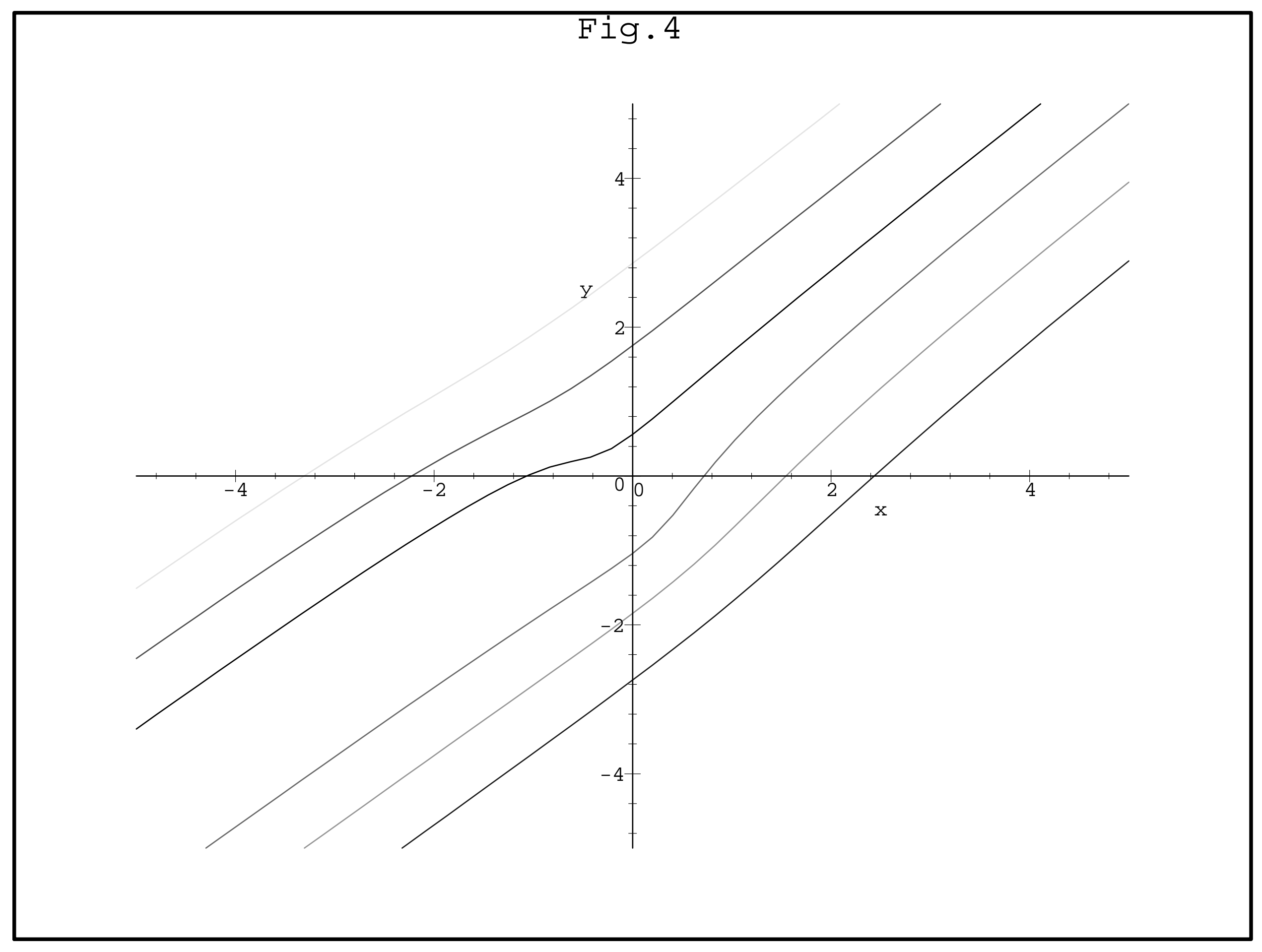




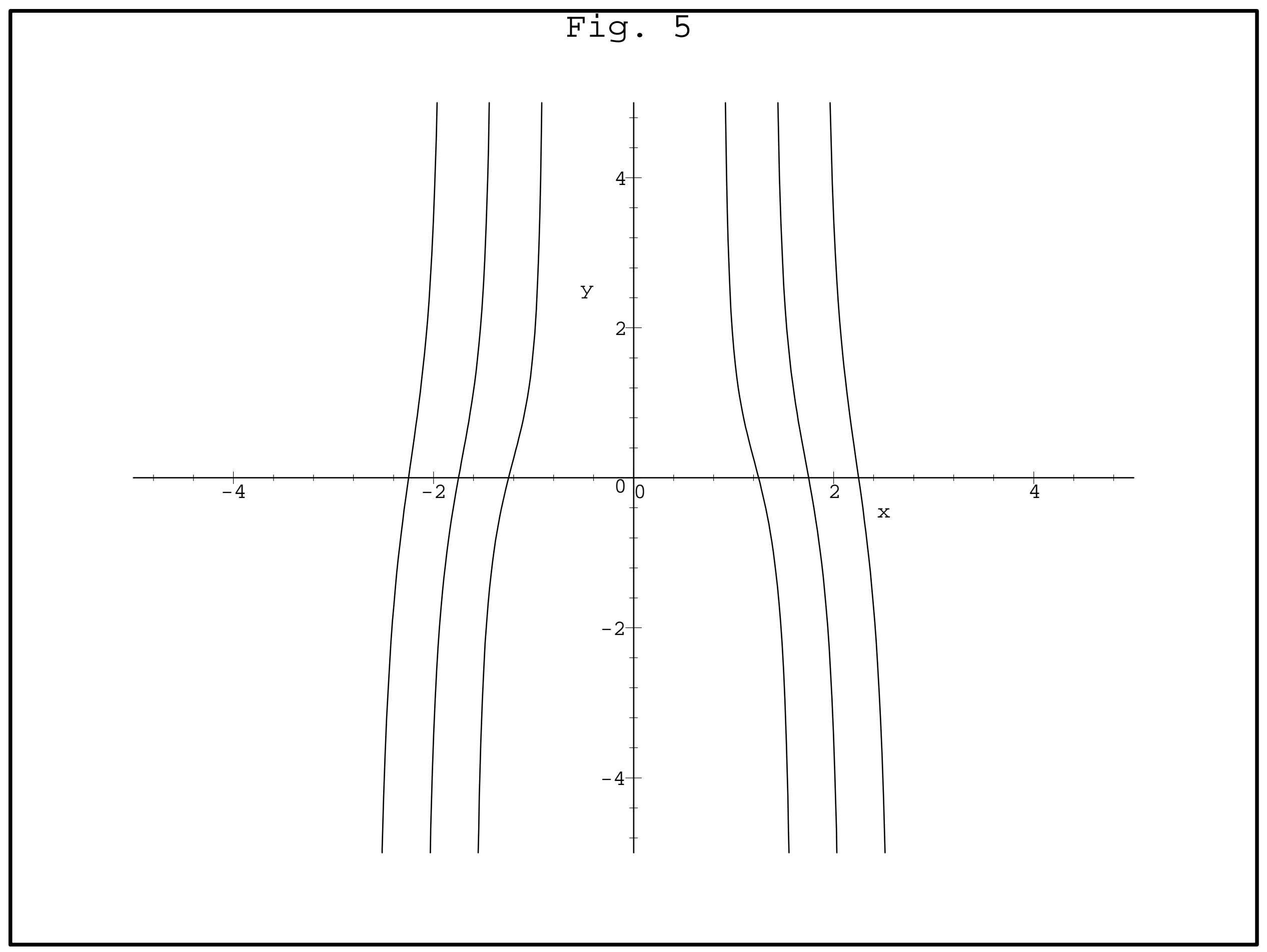

